\title{
Symptomatic approach to problems while working in the tropics
}

\author{
MJ World
}

\section{Introduction}

The best approach to a tropical deployment is to assume that diagnostic facilities will be minimal and that reliance, within reason, should be based on clinical methods (Figure 1) and so-called "near bedside" technology such as urinary and blood dipsticks (Figure 2). If additional capability is available, this bonus can be used to potential advantage but is never a substitute for careful clinical evaluation. A laboratory result which is not compliant with clinical assessment may be wrong. However, it takes considerable clinical experience to challenge a laboratory report with confidence. In western countries, clinical laboratories are subject to regulation and quality control assessment of their reliability. Licensure may be withdrawn when standards are found to be below an acceptable level. The consequence is that clinicians are only rarely confronted with an inaccurate result. This situation may not be the case in developing countries. Even though rich nations may be able to afford expensive auto-analyzers, they may fail to ensure that reagents are protected from extremes of temperature and light by leaving them exposed on the airport tarmac. Thresholds to challenge local laboratory results in such situations should be lowered, particularly if the facility does not appear clean with adequate incubation facilities and refrigeration or, where appropriate, airconditioning or heating.

In a relatively short article such as this, it is not possible to provide a comprehensive consideration of all the conditions likely to be encountered during tropical deployments. Handbooks and textbooks in compact disc

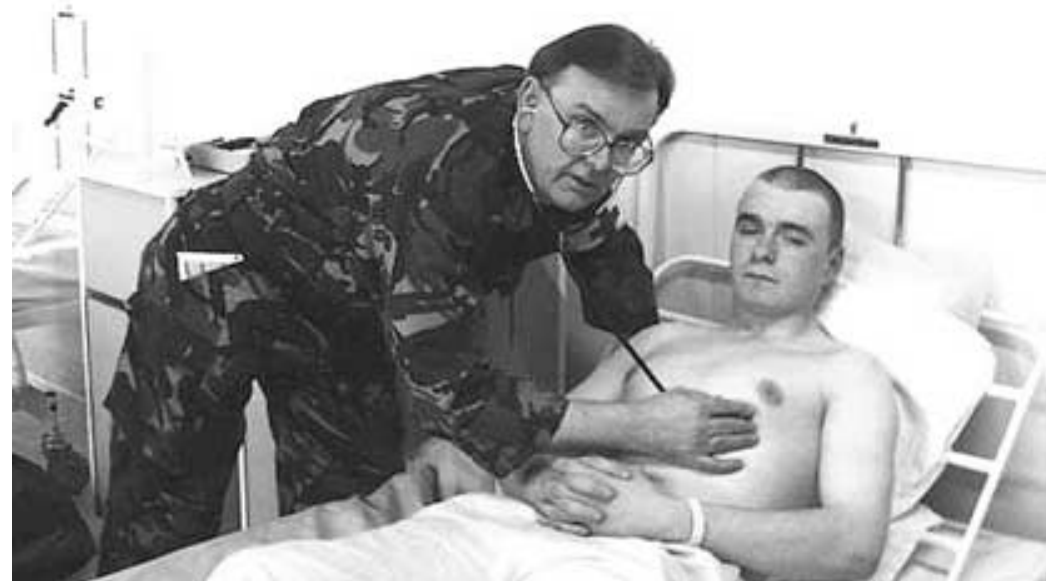

Fig 1. The author examining a patient. Considerable confidence in personal clinical assessment is essential to minimise the need for all but the most basic of investigations. format conveniently provide much more information. The emphasis here is to concentrate on common symptomatic presentations, to provide some examples of the more common tropical causes it is important to consider whilst jogging the memory of the reader of their existence and to provide a framework for evaluation and differential diagnosis. The first step is a systematic appraisal of clinical findings. This is best done by considering possible aetiologies in a logical and easilyremembered fashion. The accompanying table is an example of such an approach. Attention to detail is essential for nosological accuracy. There is no excuse for compromising successful diagnosis and treatment by an inadequate history or observation. Finally, communications are an essential part of all military deployments. In the words of a contemporary popular television quiz show, do "phone a friend" if the diagnosis is elusive: a life may depend on professional humility.

\begin{tabular}{ll} 
Table 1: Aetiologies to be considered \\
\hline Congenital: & Genetic \\
& Acquired in utero \\
Acquired: & Traumatic \\
& Infective \\
& Prions \\
& Viruses \\
& Rickettsia \\
& Bacteria \\
& Funghi \\
& Protozoa \\
& Metazo (worms) \\
& Neoplastic \\
& Cardiovascular \\
& Alimentary (including liver, gallbladder \& pancreas) \\
& Neurological \\
& Blood (haematological) \\
& Endocrine and metabolic \\
Degenerative & \\
Respiratory & Renal and genito-urinary \\
Rheumatological (including auto-immune diseases) & Drugs and toxins \\
&
\end{tabular}

Note: Acquired causes can be recalled by use of the mnemonic: TIN CAN BED 3RD

\section{History}

It is vital to try to decide which system of the body is affected. There is little potential difficulty ascribing vomiting, abdominal pain and diarrhoea to a gastro-intestinal cause but, even this could be the first manifestation of malaria or diabetes mellitus. Non-specific symptoms such as headache, nausea, myalgia and itching are much more difficult. Every symptom must be recorded. When reviewed 
in the light of subsequent developments, symptoms which were initially considered trivial may acquire such profound significance that they clinch the clinical diagnosis prompting further fruitful examination, the definitive investigation and the correct treatment.

\section{Examination}

There is no substitute for a full systemic examination. The general features of importance which frequently get forgotten are weight (a baseline indicator of nutritional and fluid status), temperature (an indicator of inflammatory, not just infective, activity), lymphadenopathy and all of the skin, not just its visible portions in the dressed patient. Most clinicians will not forget to examine the pulse or the recumbent blood pressure but many will not take the erect blood pressure to ascertain presence of postural hypotension and, hence, significant hypovolaemia which may compromise the function of vital organs, particularly the kidney. The respiratory rate is often overlooked. Tachypnoea may suggest ventilatory difficulty but the possibility of a metabolic acidosis as occurs in lactic acidosis (a common feature in severe falciparum malaria), diabetes mellitus and renal failure should be considered. While palpation and percussion are rarely omitted during abdominal examination, inspection (for abdominal distension and peristalsis) and auscultation (for bowel sounds) are often forgotten. Similarly, inspection of the external genitalia, hernial orifices and anus are all too often left out. Far too many febrile patients never have an assessment of the neck for rigidity and this omission compromises the chances of correctly diagnosing meningitis or encephalitis.

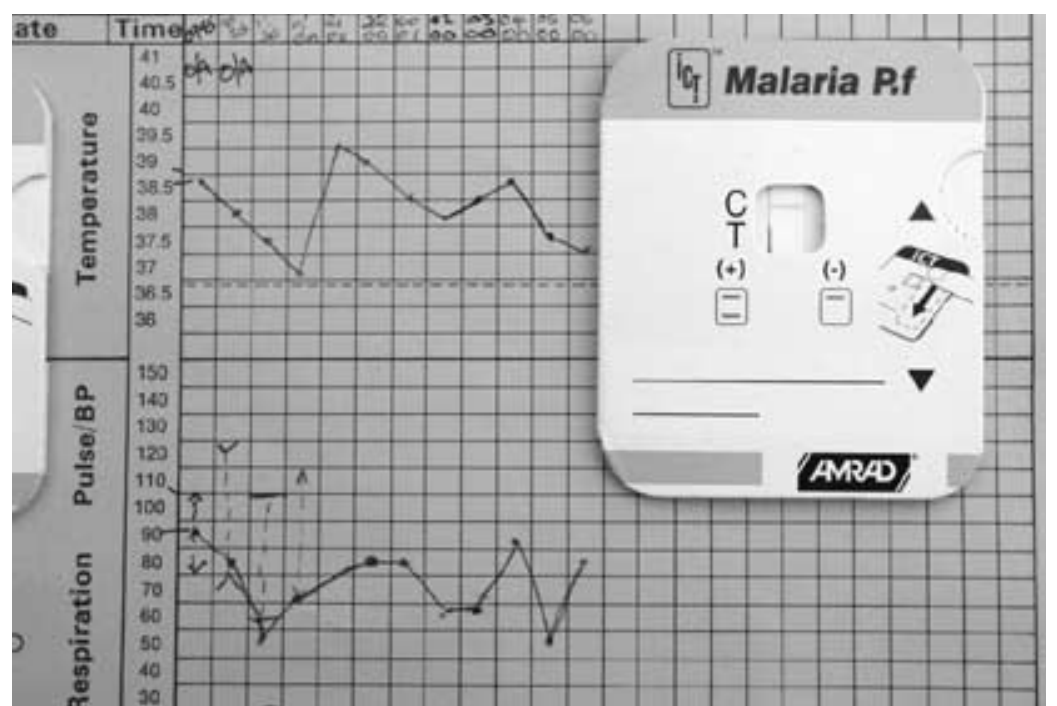

Fig 2. A negative ICT Malaria P.f ${ }^{\mathrm{TM}}$ card test for Plasmodium falciparum malaria. While this result does not exclude the possibility of other forms of malaria (eg Plasmodium vivax), it does offer considerable reassurance that the patient has not contracted the more serious form of this disease and might stimulate the military doctor to seek evidence of other causes of periodic fever. After much effort by the author to ensure its introduction, this most useful 'near bedside' test with almost $100 \%$ sensitivity and specificity is now in routine field use. (Photograph courtesy of Lt Col DF Vassallo RAMC)

\section{General Complaints}

Fever

This indicates the presence of inflammation, not just infection although the latter is probably the commonest cause. When considering infective agents, it is essential to think logically, considering micro-organisms in ascending order of size and complexity. These days one has to start with prions but, apart from the spongiform encephalopathies, no other prion-associated diseases have emerged. The old hierarchy of viruses, rickettsia, bacteria, fungi, protozoa and metazoa continues. Thinking about each of these classes of aetiological agent should ensure that such diverse diseases as viral haemorrhagic fever, leptospirosis and trichinosis are not forgotten.

Trauma, including surgery, and burns cause tissue destruction which evokes an inflammatory response. Diseases where there is an auto-immune component will often cause fever. Examples are ulcerative colitis, Crohn's disease, vasculitis (due, for example, to microscopic polyangitis or systemic lupus erythematosus) and hepatitis.

\section{Exercise intolerance}

Exercise intolerance is usually associated with easy fatigability. Very often, it is the primary complaint in the post-viral/chronic fatigue syndrome. Some care must be exercised in soldiers to determine the duration of the problem and to decide if its onset was following a major active deployment as this symptom is a prominent component of the relatively recently conceived diagnosis of post-conflict syndrome. However, a causative viral illness may be current in such conditions as anicteric hepatitis where diagnosis is almost absolutely dependent on laboratory liver function tests unless there is an increase in urinary urobilinogen in the absence of any other evidence of haemolysis. Exercise intolerance can be associated with a broad spectrum of abnormalities including anaemia, jaundice, lymphadenopathy, weight loss, dehydration and cardiac lesions.

\section{Anaemia}

Lesser degress of anaemia are difficult to detect clinically. For this reason, a portable, battery-powered haemoglobinometer is useful. In the absence of this, leaving a tube of anticoagulated blood to separate overnight permits an estimate of the haematocrit and also allows a crude assessment of the peripheral white blood cell count, an expanded buffy coat being visible if leucocytosis exists. Inspection of the serum may give clues to other problems such as hyperlipidaemia due to hypertriglyceridaemia. Of the non-nutritional tropical diseases, malaria and hookworm infestation are probably the commonest causes of anaemia. 
Faundice

Apart from yellow discolouration of the sclera, jaundice per se may be inapparent to the patient. The symptomatic presentation is very often an intolerance of smoking and alcoholic beverages with influenza-like complaints (malaise, anorexia, myalgia, fever) with itching only in the severe cases. Lesser degrees of jaundice may be difficult to detect clinically. If there is uncertainty after inspecting the inferior bulbar conjunctiva by daylight in caucasians, a part of the body which is generally protected from pigmentation by sunlight such as the abdomen should be inspected. If there is still uncertainty, it should not be forgotten that bilirubin diglucuronide is filtered at the glomerulus and concentrated in the urine giving the latter a deep orange coloration. Under active operational conditions, soldiers with definite mild jaundice who are otherwise well may have a worsening of the unconjugated hyperbilirubinaemia of Gilbert's Disease, a benign condition precipitated by fasting. The clinical clue will be absence of bilirubinuria (acholuric jaundice). Otherwise, jaundice is usually due to intrahepatic cholestasis caused by some form of hepatitis or possibly medications although causes of haemolysis such as malaria should not be forgotten. In the case of malaria, the urinary load of bilirubin diglucuronide will increase but so will that in the stools so steatorrhoea will not be present. The steatorrhoea associated with intrahepatic cholestasis should not be forgotten but may be inapparent when stools are inspected. Assuming that there has been time to immunise against hepatitis $\mathrm{A}$ and $\mathrm{B}$ viruses and no blood transfusions have been given, hepatitis $\mathrm{E}$ virus which is transmitted by the faecal-oral route, cytomegalovirus, Epstein-Barr virus or an unidentifiable hepatitis virus may have caused the hepatitis. In those who have had freshwater immersions, the possibility of leptospirosis should be considered. A neutrophil leucocytosis and elevated urea and creatinine would tend to support this diagnosis. In young soldiers, an alcoholic binge is more likely to cause acute alcohol poisoning with impaired consciousness than alcoholic hepatitis with jaundice. Laboratory liver function tests and clotting studies may permit precise monitoring of the progress of the illness but even then the clinical condition of the patient cannot be ignored. Inversion of sleep rhythm may be the earliest manifestation of hepatic encephalopathy and, although this is usually heralded by deepening jaundice, the colour of the patient may not be increased that much initially. Vigilance for the appearance of intellectual and cognitive impairments should prompt early institution of purgation, a low protein diet and gut sterilisation with relatively nonabsorbable antibiotics to limit absorption of neurotoxic amines such as octopamine. In this way, asterixis (the flapping tremor) and onset of coma should be prevented. In the absence of laboratory monitoring, the severity of urinary coloration and whole blood clotting time can be used as surrogate, imprecise assessments of progress. Return of urobilinogen in the urine heralds recovery.

\section{Lymphadenopathy}

There is a wide differential diagnosis of lymphadenopathy extending from the relatively benign such as infectious mononucleosis to the more serious lymphoproliferative disorders. AIDS-related lymphadenopathy, Lassa fever, trypanosomiasis, secondary syphilis and tuberculosis might be more commonly encountered causes in such tropical areas as West Africa. The definitive diagnosis is heavily dependent on good quality laboratory pathology. The most important step is not to miss this physical sign.

\section{Weight loss}

Any chronic debilitating disease results in weight loss but psychological problems should not be forgotten, particularly in young soldiers on their first foreign tour, especially if this is prolonged. It is essential to weigh (kilograms) the patient and to measure the height (metres) so that body mass index (BMI, $\mathrm{kg} / \mathrm{m}^{2}$ ) can be calculated. The normal BMI is $20-25 \mathrm{~kg} / \mathrm{m}^{2}$. However, the current weight has to be compared with that most recently recalled or, better, recorded. When symptoms of gastro-intestinal disturbance predominate, particularly diarrhoea, the cause of weight loss is generally immediately apparent. While neoplastic conditions are likely to be much less common in fit young soldiers, such conditions are not unknown in this age group.

\section{Dehydration}

While a poor volume pulse, dry skin, dry mouth, reduced skin turgor and enophthalmos are usually recalled as signs to look for, postural hypotension is too often forgotten. Urinary dipsticks incorporate a colorimetric estimation of specific gravity which, if greater than 1.030 (the maximum detectable) may betoken dehydration provided there are no abnormal urinary constituents such as glucose or protein. Observing urine flow gives useful information. Normal hourly urine output should be about $60 \mathrm{ml}(1 \mathrm{ml} /$ minute $)$ and if as low as $18.5 \mathrm{ml} / \mathrm{hr}$ in subsequent hours (400ml/24hrs), acute renal failure is present. Under these circumstances, oliguria should not be treated with loop diuretics! Provided blood loss is not apparent, crystalloid transfusions will be sufficient to restore left ventricular end-diastolic volume and pressure to normal and, hence, stroke volume and cardiac output. The kidneys will 
look after themselves and exercise tolerance will be restored. The cause of the dehydration will need attention and is usually obvious in soldiers. The indigenous population in tropical countries may be much more challenging and interesting, particularly in relation to endocrine causes of dehydration. There is a high prevalence of diabetes mellitus. This will cause dehydration but may remain undetected if the urine is not tested. Obstetric care is often non-existent with pregnant women delivering in isolation. Significant post partum haemorrhage is not uncommen particularly in the multigravida and, if survived, this may precipitate Sheehan's syndrome - pituitary infarction the first manifestation of which is failure of lactation. Secondary amenorrhoea follows, with relative loss of pigmentation, hypothyroidism and hypoadrenalism being late manifestations but which lower the threshold to dehydration. In these circumstances, diabetes insipidus is uncommon but not unknown and does occur more commonly in other circumstances unrelated to pregnancy. Polydipsia and polyuria with low specific gravity of the urine that responds to a test dose of vasopressin or one of its analogues usually confirms the straightforward case. Addison's disease should be suspected in the hyperpigmented, hypotensive, dehydrated local patient with hyperkalaemia and a low bicarbonate. Tuberculosis is still likely to be the cause but metastases from carcinoma of the lung are an increasingly frequent cause now that cigarette smoking has continued for two decades and more in these communities. There are atropine-like alkaloids in many tropical plants. If ingested, symptoms of atropine poisoning may occur with hallucinations and dehydration.

\section{Cardiac lesions}

In soldiers, valvular lesions and other chronic cardiac conditions will have been excluded by medical assessments before enlistment. An acute cardiac condition likely to limit exercise tolerance is viral myocarditis where there is no pain or, at worst, a dull aching sensation. Where there is pain, pericarditis or myocardial infarction should be suspected. In either case, chewing an aspirin tablet would be helpful until further diagnostic tests, primarily an electrocardiogram, can be undertaken. In the local population, chronic rheumatic valvular lesions are common and associated with progressive loss of exercise tolerance which will accelerate if infective endocarditis has supervened. Examination offers the possibility to acquaint oneself with murmurs heard infrequently in Britain these days.

\section{Specific Complaints}

Skin lesions

Self-limiting rashes are common in soldiers and probably betoken allergic conditions of one kind or another, including drugs, or viral illnesses such as dengue. Occasionally, vasculitic illnesses such as HenochSchönlein purpura can arise for the first time whilst abroad. The rash is usually confined to the legs in adult patients and may be accompanied by arthralgia and abdominal pain occasionally accompanied by a little blood in the stools and often by haematuria, although this is usually microscopic. A generalised itch in the first 24 hours after freshwater swimming may indicate exposure to schistosomal cercariae and can be accompanied by a rash. Localised skin lesions confined to exposed parts are more likely to be traumatic or the consequence of insect bites. Sometimes the insects transmit organisms other than the protozoan illnesses malaria, trypanosomiasis or leishmaniasis. In Belize, bot fly eggs are inoculated into the skin by mosquitoes. Painful development of the larvae is followed by the emergence of a maggot. Although alarming, this is not dangerous provided cutaneous bacterial infection is avoided but even then a furuncle is likely to be the worst outcome. Rickettsial illnesses cause a vasculitis and although unlikely, one of the forms of typhus should be considered. Not all are transmitted by lice but that is no reason not to look for these arthropods particularly in members of the local population. Chronic progressive ulceration suggests the possibility of cutaneous leishmaniasis and a skin biopsy is usually necessary to confirm this diagnosis. Of the metazoa, feline and canine hookworms can cause cutaneous larva migrans and strongyloides causes the similar larva currens. The serpigineous skin lesion is usually quite distinctive.

Bites from larger animals can be serious quite apart from the pain and risk of bacterial infection. Systemic inflammatory responses may require swift administration of adrenaline, hydrocortisone and antihistamines. Attack by canines demands that rabies prophylaxis is considered urgently. Antivenom administration to combat systemic effects of snake and scorpion envenomation is advisable having taken local advice about the best product to use when a polyvalent antivenom has not been provided already.

\section{Respiratory symptoms}

Asthma can arise at any time and might reasonably be expected to occur in foreign environments when personnel are exposed to a multitude of novel antigens. However, the possibility of transpulmonary migration of helminthic larvae should be considered if symptoms arise sometime after being in a tropical theatre of operations. Eosinophilia in the peripheral blood may not be particularly helpful but fever, pulmonary infiltrates on the chest X-ray and larvae in the sputum are 
highly suggestive of Loeffler's syndrome. The cause may be roundworms (ascaris), hookworms (ankylostoma, necator) or strongyloides. All of these should respond to albendazole but ivermectin, a macrolide antibiotic with antihelminthic activity, may ultimately prove to be the treatment of choice.

\section{Gastrointestinal Symptoms}

Anorexia, nausea and vomiting, abdominal pain and diarrhoea generally herald gastroenteritis. Blood loss in the stool should raise suspicion of a non-infective cause but Shigella dysenteriae, enteropathogenic Escherichia coli, amoebae and schistosomes can all cause this. In the presence of fever, malaria must always be considered. Fresh stool samples need to be examined by a formol-ether concentration technique for ova, cysts and parasites and by culture for enteropathogenic bacteria. If multiple blood smears are negative for malaria, this protozoon is unlikely to be the casue of symptoms. Even if a bacterial cause is identified, antibiotics are not always required. The gut has a complex and effective immune system which can neutralise a high proportion of infections and, in most cases of gastroenteritis, spontaneous resolution can be expected. Persisting symptoms or any element of clinical dehydration should prompt the use of a single dose of ciprofloxacin $500 \mathrm{mg}$ orally with, possibly, a further dose after 12 hours. If this fails, the possibility of protozoa (giardia, cryptosporidium, isospora and microsporidium) should be considered. A duodenal aspirate may be necessary to recover these organisms. Giardia may respond to imidazoles such a metronidazole or tinidazole. There are no drugs of proven efficacy to combat the other organisms. However, these should be eliminated by the mucosa-associated immune system provided this is not compromised. There is a high prevalence of acquired immune deficiency syndrome in African countries where water supplies are likely to be contaminated by enteric bacteria and protozoa from animals and so chronic diarrhoeas due to these causes are likely to be encountered when dealing with local patients. Profuse bloody diarrhoea in these patients should raise the question of viral haemorrhagic fever. Full protective precautions should be employed at all times. Sanitary disposal of all biological fluids, not just faeces, is essential.

In military patients with persisting gastrointestinal symptoms in the absence of identifiable pathogens, the possibility of inflammatory bowel disease - ulcerative colitis and Crohn's disease - has to be considered. These diseases usually require endoscopic evaluation to establish the diagnosis. Local facilities may be adequate but if there is any doubt at all about this and repatriation is possible within a reasonable period in relation to clinical severity, investigation is probably best delayed until the best possible facilities are available. In the interim, symptomatic treatment to control diarrhoea such as codeine phospate (this will also provide some analgesia unlike loperimide or co-phenatrope) and fluid replacement will be adequate.

Intestinal worms are unlikely to cause significant symptoms in the short term. Their presence should be considered in those with gastro-intestinal symptoms who have travelled in the tropics in the past. Evaluation of such cases is the subject of an accompanying article.

\section{Genito-urinary problems}

Haematuria

When macroscopic and occurring in the absence of trauma, this symptom usually prompts an urgent consultation. Clinical examination should include assessment of kidneys, bladder and external genitalia. Coincidence of severe unilateral colicky loin pain may suggest urolithiasis but renal swelling due to nephritis can be difficult to distinguish on occasions and the first manifestation of hitherto undiscovered polycystic kidneys where a cyst has burst must be remembered. Absence of enlarged kidneys on abdominal examination does not exclude this condition. In the febrile female patient, acute pyelonephritis has to be considered but it is unwise and bad medical practice, particularly in males, to assume that the cause of haematuria is an infection in an afebrile patient and to prescribe antibiotics without at least urinary microscopy. There are two key primary investigations. The first is to microscope the centrifuged urinary deposit. Pigmenturia (haemoglobin, myoglobin or other pigment such as beetroot) will be apparent immediately as no sedimentation of the pigment will occur. The morphology of the excreted red cells will indicate whether the cause is glomerulonephritis. Dysmorphic red cells will confirm this diagnosis whether or not red cell or other tubular casts are present. Wellhaemoglobinised undistorted red cells occur when the haematuria arises from urothelium. Excess white cells (more than 5 per high power field) can occur in renal inflammation (due to disease or due to drugs such as analgesics) but if organisms (other than Doderlein's bacilli in female patients) are visible then pyelonephritis is the cause. In schistosomiasis, eggs may be visible. In this disease, there should be a history of freshwater immersion and it is best to examine several midday samples of urine. The other key investigation is an ultrasound examination of the urinary tract. Polycystic kidneys should immediately be apparent. An inflamed kidney will be large rather than small. Small stones may be difficult to 
identify by ultrasound but a dilated collecting system should be apparent. This coupled with normal red cell morphology should prompt a request for surgical evaluation by intravenous urography and cystoscopy neither of which are required immediately. If neither urinary microscopy or ultrasound are available, antibiotics should be given to the obvious case of pyelonephritis in a female patient and pain relief for all those requiring it before referral to a unit offering the full range of nephro-urological investigations. Urothelial tumours are rare in Service personnel but are not unknown. Haematuria with pain on micturition suggests a urethral problem and, if associated with persistent hypogastric pain and possibly low back or rectal pain, prostatitis should be considered. In both cases, genito-urinary causes have to be evaluated starting with a sexual history.

\section{Symptoms of sexually transmitted infection}

Sexually transmitted infections usually manifest as penile or vulval lesions, purulent discharges or dysuria. Every effort should be made to encourage avoidance of sexual contact with the local population and the use of condoms at all times. Evaluation of these symptoms requires considerable expertise and knowledge for a diagnosis to be made with confidence as this is crucial in determining the correct treatment. Even then, infection with more than one agent is possible and although a non-specific urethritis may be certain, failure to assess serology for human immuno-deficiency virus could be viewed as medical negligence. In the absence of immediate expert assistance, the best management strategy is to take such swabs, smears and blood samples as may be appropriate and to provide symptomatic relief for urethritic symptoms with analgesics and high fluid intake with antibiotics being reserved for those symptomatically severe cases where more conservative treatments fail. No assumptions concerning diagnosis should be made and referral for assessment by a physician or a clinical nurse practitioner in genito-urinary medicine should be made at the earliest opportunity.

\section{Headache}

Acute infections such as malaria and meningitis followed by those with a more insidious onset such as brucellosis and typhoid are the conditions causing headache which should not be missed. Fortunately, brain tumours are rare in fit military personnel but not unknown. Headache worse on waking is a symptom associated with the presence of raised intracranial pressure but before making this deduction a full neurological examination is essential. Provided the increase in size of an intracranial space-occupying lesion is slow, the neurological deficit can be surprisingly minor for a tumour of some size but the neurological examination is normal only on rare occasions. Associated fever, fits, neck stiffness and disturbance of personality suggest the possibility of encephalitis or meningitis but cerebral malaria must always be considered as early treatment may be successful whereas any delay may be fatal. Blood films should be examined employing capillary blood samples. Brain imaging may not be immediately available. However, if there is no evident papilloedema and a laboratory capable of confident evaluation of cerebrospinal fluid is available, lumbar puncture by an experienced operator can be undertaken with cautious withdrawal of the stilette so that no rapid reduction of any elevated spinal pressure occurs which might precipitate coning. Turbid fluid suggests inflammation probably due to infection. Coadministration of acyclovir with antibiotics is advisable in order to cover the possibility of Herpes simplex exchphalitis. Further treatment would depend on laboratory findings and clinical developments but progressive neurological disease will require full evaluation with neurological imaging and possible electrophysiological assessment. Apart from viral and bacterial infections, tropical causes of neurological abnormality in caucasians are few and rare. Trypanosomiasis arising in east Africa (socalled Rhodesian variant) can present as a fulminant meningo-encephalitis but this is more common in children. Cysticercosis commonly presents as a late-onset fit in Gurkhas. Hydatid cysts and schistosomal eggs can occur rarely in the central nervous system. More common than any of these tropical conditions but still rare is GuillainBarre syndrome (acute ascending polyradiculopathy). The herald symptom is frequently ascending hypoaesthesia in a stocking distribution. There may be no convincing objective sensory deficit. Observation is essential to determine outcome and progression with development of weakness should be treated with concern to ensure facilities for ventilation are available.

\section{Locomotor symptoms}

In caucasians, non-traumatic muscular and joint pains commonly accompany many infections, the majority of them being viral and minor. Symptomatic treatment with non-steroidal inflammatory drugs is often all that is required. Even in dengue fever, also known as break bone fever on account of its symptomatic severity, adequate analgesia is the only management option. In many tropical countries serious rheumatological conditions such as systemic lupus erythematosus and Behcet's disease are much more prevalent in the indigenous population. However, initial attacks of these conditions can arise in caucasians when 
abroad and should not be dismissed from the differential diagnosis as they are erroneously considered impossible. In older caucasian males, microscopic polyangitis should be considered when there is fever with eosinophilia yet helminthic infection cannot be demonstrated.

\section{Conclusion}

Symptoms arising in soldiers during tropical deployments should never be dismissed out of hand. Soldiers generally have a high threshold for complaint and seek medical advice only reluctantly. The diagnosis may not be immediately evident but even at this early stage could be potentially lifethreatening. A period of observation will be reassuring whilst giving time to consider and to discuss with a more experienced colleague some of the serious conditions mentioned here. 